\title{
SYNTHESIS, CHARACTERIZATION AND IN VITRO ANTIMICROBIAL EVALUATION OF SOME NOVEL BENZIMIDAZOLE DERIVATIVES BEARING HYDRAZONE MOIETY
}

\author{
JAYANTA SARMA, GURVINDER SINGH, MUKTA GUPTA, REENA GUPTA, BHUPINDER KAPOOR*
}

Department of Pharmaceutical Chemistry, School of Pharmaceutical Sciences, Lovely Professional University, Phagwara - 144411, Punjab, India. Email: bhupinder.14146@Ipu.co.in

Received: 14 July 2017, Revised and Accepted: 25 July 2017

\begin{abstract}
Objective: The synthesis of novel benzimidazole-hydrazone derivatives has been carried out based on the previous findings that both these pharmacophores possess potent antimicrobial activities. The antibacterial properties of synthesized derivatives were screened against both Grampositive and Gram-negative bacteria.
\end{abstract}

Methods: $O$-phenylenediamine on condensation with substituted aromatic acids in polyphosphoric acid gave benzimidazole nucleus which on reaction with ethyl chloroacetate and hydrazine hydrate in two different steps resulted in the formation of substituted acetohydrazides. The targeted compounds 6a-l were synthesized by reaction of substituted acetohydrazides with aromatic aldehydes and screened for their antibacterial potential by cup-plate method.

Results: The synthesized benzimidazole-hydrazones exhibited moderate to strong antibacterial activities against Staphylococcus aureus, Bacillus subtilis, Escherichia coli, and Pseudomonas aeruginosa. The compounds $6 \mathrm{a}-6 \mathrm{f}$ were found to be most effective against $S$. aureus, E. coli, and P. aeruginosa. Among all the synthesized compounds, the zone of inhibition of $6 \mathrm{f}$ in highest concentration, i.e., $100 \mu \mathrm{g} / \mathrm{ml}$ were found to be $>31 \mathrm{~mm}$ against all the stains of bacteria.

Conclusion: The antibacterial results revealed that the synthetized derivatives have significant antimicrobial properties and further structure activity relationship studies may develop more potent and less toxic molecules.

Keywords: Benzimidazole, Hydrazone, Antimicrobial, Antibacterial.

(C) 2017 The Authors. Published by Innovare Academic Sciences PvtLtd. This is an open accessarticle under the CCBY license (http://creativecommons. org/licenses/by/4. 0/) DOI: http://dx.doi.org/10.22159/ajpcr.2017.v10s4.21328

\section{INTRODUCTION}

Resistance to commercially available antimicrobial agents such as $\beta$-lactam antibiotics, quinolones, and macrolides is a major health problem worldwide [1]. Infections caused by multidrug-resistant microorganisms are responsible for significant increase in morbidity and mortality, prolonged treatment period, and increased healthcare cost [2]. Due to seriousness of this problem, the World Health Organisation in 2011 has selected "antimicrobial resistance: No action today no cure tomorrow" as the theme for World Health Day [3,4].

One way to counteract the challenge of microbial resistance is judicious use of currently available antibiotics; the other is the development of new anti-ineffective agents with enhanced activity and a novel mechanism of action $[5,6]$. The bacteria exhibit phenotypic resistance, i.e., tolerance, rendering them to 1000 -fold more resistant to commercially available antibiotics, is due to their ability to grow in sessile or adherent state which leads to formation of biofilms [7].

Benzimidazole is an important pharmacophore and privileged structure in the field of medicinal chemistry because of its varied biological activities, viz., anticancer [8,9], antihypertensive [10,11], antiviral [12,13], anti-inflammatory [14,15], antihistaminic [16,17], antiulcer [18], anticoagulant $[19,20]$, and antimicrobial [21,22]. The antimicrobial action of benzimidazole is due to its structural similarity to purines; therefore, its derivatives inhibit nucleic acid and protein synthesis by competing with natural purines; thereby inhibit the growth as well as kill bacterial strains [23].

Hydrazones constitute another important class of pharmacologically active drug molecules which has attracted the attention of medicinal chemists due to their diverse biological activities such as analgesic, anthelmintic, anticonvulsant, antidepressant, anti-inflammatory, antimalarial, anticancer, antiviral, and antibacterial [24]. Some commercially available antibacterial drugs such as furacilin, furazolidone, ftivazide, nifuroxazide, nirofurazone, and nitrofurantoin are known to contain hydrazone group [25-27].

Due to the chemotherapeutic potential of both benzimidazole and hydrazone compounds, it was hypothesized that it would be worthwhile to synthesize novel molecules having both these pharmacophores in a single chemical entity. Furthermore, extensive literature search also suggested that only a limited efforts have been made to combine both these vital moieties in a single scaffold $[1,28]$. These findings boosted us to synthesis novel benzimidazole-hydrazone derivatives as antimicrobial agents.

\section{METHODS}

Chemistry

The chemicals used in the synthesis were procured from Merck, SigmaAldrich, and Loba Chemie, India. All the solvents were of commercial grade and distilled before use. Open capillary method was used to determine the melting points (m.p) of the synthesized derivatives using digital melting point apparatus (Popular, India). The progress of reactions was monitored by thin layer chromatography on silica gel $\mathrm{F}_{254}$ plates with visualization by ultraviolet or iodine vapors. The molecular structures of all the synthesized derivatives were confirmed by elemental analysis, infrared (IR), ${ }^{1} \mathrm{H}$ nuclear magnetic resonance (NMR) as well as mass spectra. The IR spectra (in $\mathrm{KBr}$ pellet) were recorded on FTIR-8400S (Shimadzu) spectrophotometer. Bruker 
Avance II (400 MHz) spectrometer was used to record ${ }^{1} \mathrm{H}$ NMR and chemical shifts were given in $\delta(\mathrm{ppm})$ scale. Mass spectra were obtained with Waters Q-Tof micromass spectrometer, and elemental analysis was performed on a Thermo Flash 2000 analyzer.

The general strategy for synthesis of benzimidazole-hydrazone derivatives is illustrated in Scheme 1.

\section{Experimental}

Synthesis of 2-(substituted phenyl)-1H-benzimidazole 3a-d

An equimolar quantities of $o$-phenylenediamine $1(0.05 \mathrm{~mol})$ and substituted benzoic acid 2a-d $(0.05 \mathrm{~mol})$ were dissolved in poly phosphoric acid $(15 \mathrm{~g})$ and refluxed for $6-9$ hrs at $180-185^{\circ} \mathrm{C}$ with constant stirring. The reaction mixtures were cooled and slowly poured into $200 \mathrm{~g}$ of crushed ice with constant stirring. The $\mathrm{pH}$ of the reaction mixtures were adjusted to alkaline by adding $4 \mathrm{~N}$ of anhydrous sodium carbonate. The precipitated solids were filtered under pressure, washed with distilled water and recrystallized from ethanol $[29,30]$.

\section{2-Phenyl-benzimidazole $3 a$}

IR (KBr, cm-1): 3404 (N-H sec.), 3047 (C-H str. Ar), 1664 (N=C), 1591 and $1462(\mathrm{C}=\mathrm{C}), 1276(\mathrm{C}-\mathrm{N}), 750$ and 690 (mono subst. oop); ${ }^{1} \mathrm{H}$ NMR (DMSO- $\left.d_{6}, 400 \mathrm{MHz}\right) \delta: 7.17-7.21(\mathrm{t}, 2 \mathrm{H}$, benzimidazole), 7.40-7.50 (m, $3 \mathrm{H}$, phenyl), 7.60-7.68 (d, $2 \mathrm{H}$, phenyl), 8.20-8.22 (d, $2 \mathrm{H}$, benzimidazole), 12.83 (s, NH); ES-MS (m/z): $195[\mathrm{M}+1]$; Anal. calcd for $\mathrm{C}_{13} \mathrm{H}_{10} \mathrm{~N}_{2}$ : C, 80.39; H, 5.19; N, 14.42. Found: C, 80.21; H, 5.20; N, 14.59 .

\section{2-(2-Hydroxyphenyl)-1H-benzimidazole $3 b$}

IR ( $\mathrm{KBr}, \mathrm{cm}^{-1}$ ): 3321 (N-H sec.), $3225(\mathrm{O}-\mathrm{H}), 3070$ (C-H str. Ar), 1626 $(\mathrm{N}=\mathrm{C}), 1606$ and 1489 (C=C), 1246 (C-N), 750 (ortho subst. oop); ${ }^{1} \mathrm{H}$ NMR (DMSO- $\left.d_{6}, 400 \mathrm{MHz}\right) \delta$ : 6.88-6.90 (t, 1H, 2-hydroxyphenyl), 6.956.97 (d, 1H, 2-hydroxyphenyl), 7.19-7.21 ( $\mathrm{m}, 2 \mathrm{H}$, benzimidazole), 7.277.29 (t, 1H, 2-hydroxyphenyl), 7.55-7.56 (s, 1H, OH), 7.93-7.95 (d, 2H, benzimidazole), 12.74 (s, NH); ES-MS (m/z): 211 [M+1]; Anal. calcd for $\mathrm{C}_{13} \mathrm{H}_{10} \mathrm{~N}_{2} \mathrm{O}: \mathrm{C}, 74.27 ; \mathrm{H}, 4.79 ; \mathrm{N}, 13.33$. Found: $\mathrm{C}, 74.21 ; \mathrm{H}, 4.72 ; \mathrm{N}, 13.49$.

\section{2-(2-Chlorophenyl)-1H-benzimidazole $3 c$}

IR ( $\mathrm{KBr}, \mathrm{cm}^{-1}$ ): 3061 ( $\mathrm{N}-\mathrm{H} \mathrm{sec}$.), 3047 (C-H str. Ar), 1589 \& 1442 (C=C), 1317 (C-N), 750 (ortho subst. oop); ${ }^{1} \mathrm{H}$ NMR ( $\mathrm{CDCl}_{3}, 400 \mathrm{MHz}$ ) $\delta: 7.28-$ 7.37 (m, 2H, benzimidazole), 7.40-7.48 (m, 2H, 2-chlorophenyl), 7.517.54 (dd, 1H, 2-chlorophenyl), 7.71-7.73 (m, 2H, benzimidazole), 8.458.48 (dd, 1H, 2-chlorophenyl), 12.73 (s, NH); ES-MS (m/z): 229 [M+1]; Anal. calcd for $\mathrm{C}_{13} \mathrm{H}_{9} \mathrm{~N}_{2} \mathrm{Cl}$ : C, 68.28; $\mathrm{H}, 3.97 ; \mathrm{N}, 12.25$. Found: C, 68.34; $\mathrm{H}$, 3.91; N, 12.27

\section{2-(3-Chlorophenyl)-1H-benzimidazole $3 d$}

IR (KBr, cm${ }^{-1}$ ): 3045 (N-H sec.), 1541 and 1442 (C=C), 1317 (C-N), 895, 744 and 680 (meta subst. oop); ${ }^{1} \mathrm{H}$ NMR (DMSO- $d_{6}, 400 \mathrm{MHz}$ ) $\delta:$ 7.17-7.17 (dd, $2 \mathrm{H}$, benzimidazole), 7.39-7.41 (m, 2H, 3-chlorophenyl), 7.52-7.53 (m, $2 \mathrm{H}$, benzimidazole), 8.08-8.11 (d, 1H, 3-chlorophenyl), 8.20-8.21 (s, $1 \mathrm{H}$, 3-chlorophenyl), 12.83 (s, NH); ES-MS ( $\mathrm{m} / \mathrm{z}$ ): 229 [M+1]; Anal. calcd for $\mathrm{C}_{13} \mathrm{H}_{9} \mathrm{~N}_{2} \mathrm{Cl}$ : C, 68.28; $\mathrm{H}, 3.97 ; \mathrm{N}, 12.25$. Found: $\mathrm{C}, 68.37 ; \mathrm{H}, 3.84 ; \mathrm{N}, 12.24$.

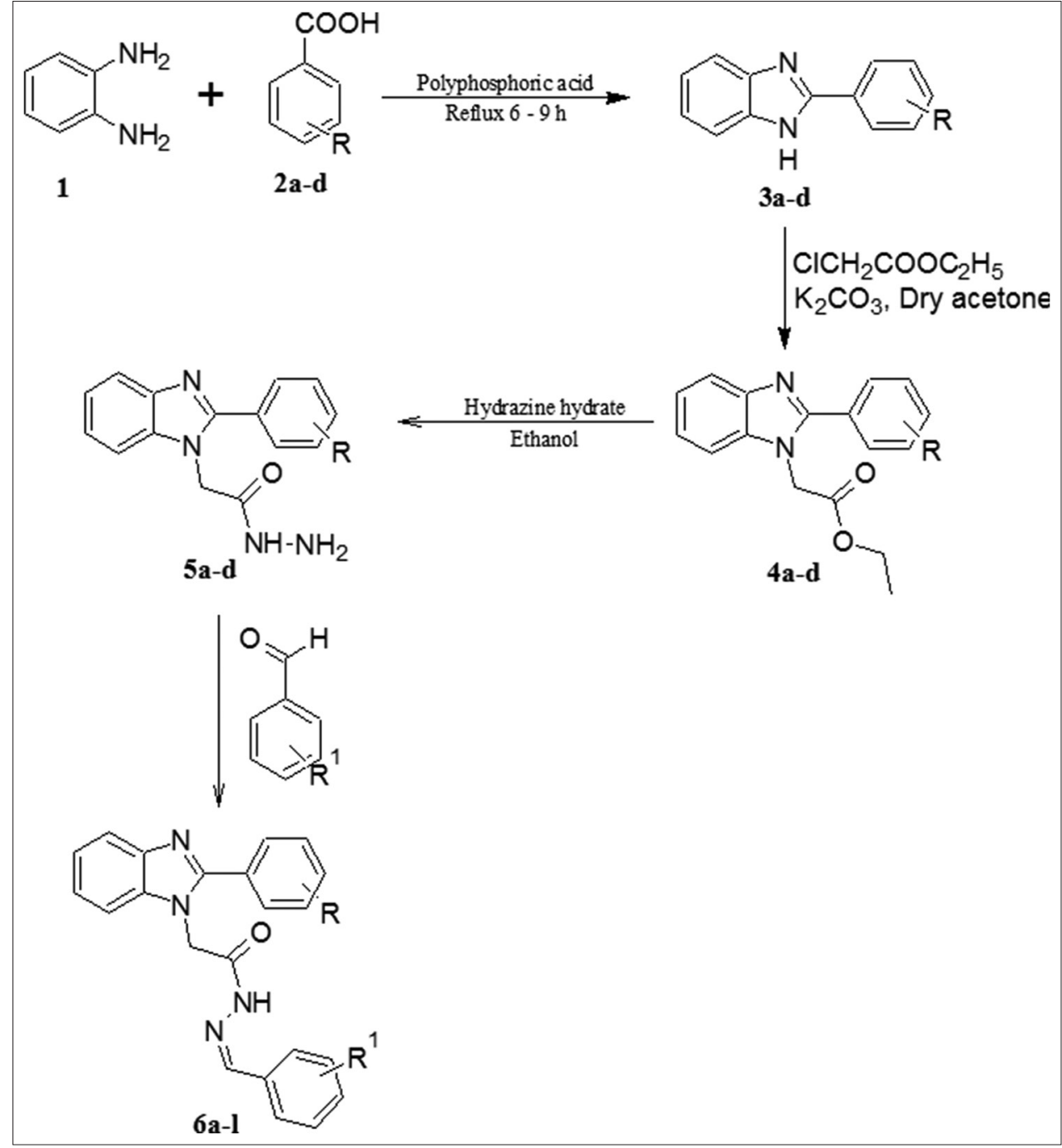

Scheme 1: Synthesis of benzimidazole-hydrazone derivatives 6a-1 
Synthesis of ethyl-2-[2-(substituted phenyl)-1H-benzimidazol-1yl]acetate $4 a-d$

Ethylchloroacetate $(0.01 \mathrm{~mol})$ was added to a solution of 2-(substituted)$1 \mathrm{H}$-benzimidazole $3 \mathrm{a}-\mathrm{d}(0.01 \mathrm{~mol})$ in dry acetone $(40 \mathrm{ml})$, followed by addition of anhydrous potassium carbonate $(2 \mathrm{~g})$. The reaction mixture was refluxed for 10-12 hrs. The solvent was removed under vacuum by rotary evaporator and the residue was recrystallized from ethanol to give $4 a-d$ [31-33].

\section{Ethyl-2-(2-phenyl-1H-benzimidazol-1-yl)acetate $4 a$}

IR ( $\mathrm{KBr}, \mathrm{cm}^{-1}$ ): 3047 (C-H str. ArH), 1742 (C=0), $1683(\mathrm{C}=\mathrm{N}), 1622$ and 1462 (C=C), 1276 (C-N), 1226 (C-O-C), 742 and 704 (mono subst. oop); ${ }^{1} \mathrm{H}$ NMR (DMSO- $\left.d_{6}, 400 \mathrm{MHz}\right) \delta: 1.18\left(\mathrm{~m}, 3 \mathrm{H}, \mathrm{CH}_{3}\right), 4.3\left(\mathrm{~m}, 2 \mathrm{H}, \mathrm{CH}_{2}\right)$, $4.7\left(\mathrm{~m}, 2 \mathrm{H}, \mathrm{OCH}_{2}\right)$ 7.13-7.17 (m, 2H, benzimidazole), 7.36-7.45 (m, 3H, phenyl), 7.53-7.56 (m, 2H, phenyl), 8.12-8.14 (d, $2 \mathrm{H}$, benzimidazole); ES-MS (m/z): $281[\mathrm{M}+1]$; Anal. calcd for $\mathrm{C}_{17} \mathrm{H}_{16} \mathrm{~N}_{2} \mathrm{O}_{2}: \mathrm{C}, 72.84 ; \mathrm{H}, 5.75 ; \mathrm{N}$, 9.99. Found: C, 72.89; H, 5.84; N, 9.76.

Ethyl-2-\{2-(2-hydroxyphenyl)-1H-benzimidazol-1-yl\}acetate $4 b$ IR $\left(\mathrm{KBr}, \mathrm{cm}^{-1}\right)$ : $3246(\mathrm{O}-\mathrm{H}), 3051$ (C-H str. $\left.\mathrm{ArH}\right), 1735(\mathrm{C}=\mathrm{O})$, $1629(\mathrm{C}=\mathrm{N}), 1589$ and $1417(\mathrm{C}=\mathrm{C}), 1274(\mathrm{C}-\mathrm{N}), 1250$ (C-O-C), 746 (ortho subst. oop); ${ }^{1} \mathrm{H}$ NMR (DMSO- $\left.d_{6}, 400 \mathrm{MHz}\right) \delta: 1.19(\mathrm{~m}$, $\left.3 \mathrm{H}, \mathrm{CH}_{3}\right), 4.16\left(\mathrm{~m}, 2 \mathrm{H}, \mathrm{CH}_{2}\right), 4.7\left(\mathrm{~m}, 2 \mathrm{H}, \mathrm{OCH}_{2}\right), 7.14-7.19(\mathrm{~m}, 2 \mathrm{H}$, benzimidazole), 7.38-7.48 (m, 4H, 2-hydroxyphenyl), 7.53-7.56 (m, $2 \mathrm{H}$, benzimidazole), 7.9-8.0 (s, 1H, OH); ES-MS ( $\mathrm{m} / \mathrm{z}): 297$ [M+1]; Anal. calcd for $\mathrm{C}_{17} \mathrm{H}_{16} \mathrm{~N}_{2} \mathrm{O}_{3}$ : C, 68.91; $\mathrm{H}, 5.44 ; \mathrm{N}, 9.45$. Found: C, 68.76; H, 5.36; N, 9.38 .

Ethyl-2-\{2-(2-chlorophenyl)-1H-benzimidazol-1-yl\}acetate 4c IR ( $\left.\mathrm{KBr}, \mathrm{cm}^{-1}\right)$ : 3061 (C-H str. ArH), 2966 (C-H str. Aliphatic), 1749 (C=0), $1683(\mathrm{C}=\mathrm{N}), 1622$ \& 1489 (C=C), 1315 (C-N), 1053 (C-O-C), 742 (C-Cl); ${ }^{1} \mathrm{H}$ NMR (DMSO- $\left.d_{6}, 400 \mathrm{MHz}\right) \delta: 1.20\left(\mathrm{~m}, 3 \mathrm{H}, \mathrm{CH}_{3}\right), 4.23\left(\mathrm{~m}, 2 \mathrm{H}, \mathrm{CH}_{2}\right)$, $4.9\left(\mathrm{~m}, 2 \mathrm{H}, \mathrm{OCH}_{2}\right), 7.16-7.20(\mathrm{~m}, 2 \mathrm{H}$, benzimidazole $), 7.37-7.44(\mathrm{~m}, 2 \mathrm{H}$, 2-chlorophenyl), 7.51-7.52 (m, 1H, 2-chlorophenyl), 7.58-7.61 (m, 2H, benzimidazole), 7.88-7.92 (m, 1H, 2-chlorophenyl); ES-MS ( $\mathrm{m} / \mathrm{z}): 315$ $[\mathrm{M}+1]$; Anal. calcd for $\mathrm{C}_{17} \mathrm{H}_{15} \mathrm{~N}_{2} \mathrm{O}_{2} \mathrm{Cl}$ : C, 64.87; $\mathrm{H}, 4.80 ; \mathrm{N}, 8.90$. Found: $\mathrm{C}$, 64.64; H, 4.92; N, 8.86.

\section{Ethyl-2-\{2-(3-chlorophenyl)-1H-benzimidazol-1-yl\}acetate $4 d$} IR ( $\mathrm{KBr}, \mathrm{cm}^{-1}$ ): 3045 (C-H str. ArH), 2964 (C-H str. aliphatic), 1749 (C=0), $1683(\mathrm{C}=\mathrm{N}), 1602$ \& 1471 (C=C), 1317 (C-N), 1124 (C-O-C), 744 (C-Cl); ${ }^{1} \mathrm{H}$ NMR (DMSO- $\left.d_{6}, 400 \mathrm{MHz}\right) \delta: 1.19\left(\mathrm{~m}, 3 \mathrm{H}, \mathrm{CH}_{3}\right), 4.21\left(\mathrm{~m}, 2 \mathrm{H}, \mathrm{CH}_{2}\right)$, $4.83\left(\mathrm{~m}, 2 \mathrm{H}, \mathrm{OCH}_{2}\right), 7.14-7.19(\mathrm{~m}, 2 \mathrm{H}$, benzimidazole $), 7.38-7.47(\mathrm{~m}, 2 \mathrm{H}$, 3-chlorophenyl), 7.53-7.56 (m, 2H, benzimidazole), 8.07-8.09 (d, $1 \mathrm{H}$, 3-chlorophenyl), 8.19-8.20 (s, 1H, 3-chlorophenyl); ES-MS (m/z): 315 $[\mathrm{M}+1]$; Anal. calcd for $\mathrm{C}_{17} \mathrm{H}_{15} \mathrm{~N}_{2} \mathrm{O}_{2} \mathrm{Cl}$ : C, 64.87; $\mathrm{H}, 4.80 ; \mathrm{N}, 8.90$. Found: $\mathrm{C}$, 64.69; H, 4.98; N, 8.84.

Synthesis of 2-[2-(substituted phenyl)-1H- benzimidazol-1-yl] acetohydrazide 5 a-d

Hydrazine hydrate $(0.01 \mathrm{~mol})$ was added to ethanolic solution of ethyl [2-(substituted phenyl)-1H-benzimidazol-1-yl]acetate $4 \mathrm{a}-\mathrm{d}$ and the reaction mixture was refluxed for 3-4 hrs. After the completion of reaction, the mixture was cooled in ice bath; the solid obtained was filtered, washed with cold water and recrystallized from methanol.

\section{2-(2-Phenyl-1H-benzimidazol-1-yl)acetohydrazide $5 a$}

IR ( $\mathrm{KBr}, \mathrm{cm}^{-1}$ ): 3479 and 3416 (N-H prim.), 3047 (C-H str. ArH), 2986 (C-H str. aliphatic), 1618 (C=0), $1591 \& 1475$ (C=C), 1276 (C-N), 738 and 704 (mono subst. oop); ${ }^{1} \mathrm{H}$ NMR (DMSO- $d_{6}, 400 \mathrm{MHz}$ ) $\delta: 2.51(\mathrm{~s}, 2 \mathrm{H}$, $\left.\mathrm{NH}_{2}\right), 4.95\left(\mathrm{~s}, 2 \mathrm{H}, \mathrm{CH}_{2}\right), 7.13-7.17(\mathrm{~m}, 2 \mathrm{H}$, benzimidazole), 7.38-7.47 (m, $3 \mathrm{H}$, phenyl), 7.53-7.56 (m, 2H, phenyl), 8.13-8.15 (d, 2H, benzimidazole), 9.5 (1H, CONH); ES-MS (m/z): 267 [M+1]; Anal. calcd for $\mathrm{C}_{15} \mathrm{H}_{14} \mathrm{~N}_{4} \mathrm{O}: \mathrm{C}$, 67.65; H, 5.30; N, 21.04. Found: C, 67.58; H, 5.19; N, 20.92.
2-\{2-(2-Hydroxyphenyl)-1H-benzimidazol-1-yl\}acetohydrazide $5 b$ IR (KBr, cm${ }^{-1}$ ): 3481 and 3414 (N-H prim.), $3236(\mathrm{O}-\mathrm{H}), 3057$ (C-H str. ArH), $1618(\mathrm{C}=0), 1616$ \& $1489(\mathrm{C}=\mathrm{C}), 1259(\mathrm{C}-\mathrm{N}), 738$ (ortho subst. oop); ${ }^{1} \mathrm{H}$ NMR (DMSO- $\left.d_{6}, 400 \mathrm{MHz}\right) \delta: 2.50-2.51\left(\mathrm{~s}, 2 \mathrm{H}, \mathrm{NH}_{2}\right.$ ), $4.85\left(\mathrm{~s}, 2 \mathrm{H}, \mathrm{CH}_{2}\right), 6.88-6.96(\mathrm{~m}, 2 \mathrm{H}$, benzimidazole), 7.16-7.21 (m, 3H, 2-hydroxyphenyl), 7.27-7.29 (t, 1H, 2-hydroxyphenyl), 7.55 (s, 2H, benzimidazole), 7.94-7.96 (s, $1 \mathrm{H}, \mathrm{OH}), 9.0(1 \mathrm{H}, \mathrm{CONH})$; ES-MS $(\mathrm{m} / \mathrm{z})$ : 283 [M+1]; Anal. calcd for $\mathrm{C}_{15} \mathrm{H}_{14} \mathrm{~N}_{4} \mathrm{O}_{2}: \mathrm{C}, 63.82 ; \mathrm{H}, 5.00 ; \mathrm{N}, 19.85$. Found: C, 63.89; H, 5.4; N, 19.42 .

2-\{2-(2-Chlorophenyl)-1H-benzimidazol-1-yl\}acetohydrazide 5c IR ( $\mathrm{KBr}, \mathrm{cm}^{-1}$ ): 3481 and 3416 (N-H prim.), 3061 (C-H str. ArH), 2916 (C-H str. aliphatic), 1616 (C=0), $1591 \& 1442(\mathrm{C}=\mathrm{C}), 1232(\mathrm{C}-\mathrm{N}), 742$ (C-Cl), 738; ${ }^{1} \mathrm{H}$ NMR (DMSO- $\left.d_{6}, 400 \mathrm{MHz}\right) \delta: 2.50-2.52\left(\mathrm{~s}, 2 \mathrm{H}, \mathrm{NH}_{2}\right.$ ), $4.78\left(\mathrm{~s}, 2 \mathrm{H}, \mathrm{CH}_{2}\right), 7.16-7.20(\mathrm{~m}, 2 \mathrm{H}$, benzimidazole), 7.36-7.43 (m, $2 \mathrm{H}$, 2-chlorophenyl), 7.49-7.50 (m, 1H, 2-chlorophenyl), 7.57-7.61 (m, 2H, benzimidazole), 7.88-7.90 (m, 1H, 2-chlorophenyl), 8.98 (1H, CONH); ES-MS (m/z): 301 [M+1]; Anal. calcd for $\mathrm{C}_{15} \mathrm{H}_{13} \mathrm{~N}_{4} \mathrm{OCl}$ : C, 59.91; $\mathrm{H}, 4.36$; $\mathrm{N}, 18.63$. Found: C, 59.85; H, 4.42; N, 18.75.

2-\{2-(3-Chlorophenyl)-1H-benzimidazol-1-yl\}acetohydrazide $5 d$ IR ( $\mathrm{KBr}, \mathrm{cm}^{-1}$ ): 3481 and 3414 (N-H prim.), 3045 (C-H str. ArH), 2918 (C-H str. aliphatic), $1616(\mathrm{C}=0), 1570$ and $1462(\mathrm{C}=\mathrm{C}), 1228(\mathrm{C}-\mathrm{N})$, 742 (C-Cl), 744; ${ }^{1} \mathrm{H}$ NMR (DMSO- $\left.d_{6}, 400 \mathrm{MHz}\right) \delta: 2.50-2.51\left(\mathrm{~s}, 2 \mathrm{H}, \mathrm{NH}_{2}\right.$ ), $4.92\left(\mathrm{~s}, 2 \mathrm{H}, \mathrm{CH}_{2}\right), 7.15-7.19(\mathrm{~m}, 2 \mathrm{H}$, benzimidazole), 7.40-7.48 (m, $2 \mathrm{H}$, 3-chlorophenyl), 7.54-7.56 (m, 2H, benzimidazole), 8.08-8.10 (d, $1 \mathrm{H}$, 3-chlorophenyl), 8.19-8.20 (s, 1H, 3-chlorophenyl), $9.26(1 \mathrm{H}, \mathrm{CONH})$; ES-MS $(\mathrm{m} / \mathrm{z}): 301[\mathrm{M}+1]$; Anal. calcd for $\mathrm{C}_{15} \mathrm{H}_{13} \mathrm{~N}_{4} \mathrm{OCl}$ : C, 59.91; $\mathrm{H}, 4.36$; $\mathrm{N}, 18.63$. Found: C, 59.82; H, 4.53; N, 18.72.

Synthesis of N-(Substituted benzylidene)-2-[(2-(substituted phenyl)-1H-benzimidazol-1-yl) acetohydrazide 6a-l

A mixture of 2-[2-(substituted phenyl)- $1 \mathrm{H}$ - benzimidazol-1-yl] acetohydrazide $5 \mathrm{a}-\mathrm{d}(0.0025 \mathrm{~mol})$, substituted benzaldehyde $(0.0025$ mol) and glacial acetic acid (few drops) was refluxed for 5 hrs in ethanol $(20 \mathrm{ml})$. After the completion of reaction, the solvent was removed by rotary evaporator and the reaction mixture was cooled and poured in ice-cold water. The precipitates obtained were filtered, dried and recrystallized from ethanol to give benzimidazole-hydrazone derivatives.

$N$-Benzylidene-2-[2-(phenyl)-1H-benzimidazol-1-yl] acetohydrazide $6 a$

IR (KBr, cm${ }^{-1}$ ): 3117 (N-H sec.), 3047 (C-H str. ArH), 1668 (C=0), 1622 and $1462(\mathrm{C}=\mathrm{C}), 1224(\mathrm{C}-\mathrm{N}), 744$ and 680 (mono subst. oop); ${ }^{1} \mathrm{H}$ NMR (DMSO- $\left.d, 400 \mathrm{MHz}\right) \delta: 4.62\left(\mathrm{~s}, 2 \mathrm{H}, \mathrm{CH}_{2}\right), 7.15-7.17(\mathrm{~m}, 2 \mathrm{H}$, benzimidazole), 7.38-7.46 (m, 6H, phenyl), 7.54-7.56 (m, 4H, phenyl), 8.11-8.14 (d, 2H, benzimidazole), $8.65(\mathrm{~s}, 1 \mathrm{H}, \mathrm{N}=\mathrm{CH}), 11.07$ (1H, CONH); ES-MS (m/z): 355 [M+1]; Anal. calcd for $\mathrm{C}_{22} \mathrm{H}_{18} \mathrm{~N}_{4} \mathrm{O}: \mathrm{C}, 74.56$; $\mathrm{H}, 5.12$; N, 15.81. Found: C, 74.42; H, 5.19; N, 15.89 .

$N$-(2-Hydroxybenzylidene)-2-[2-(phenyl)-1H-benzimidazol-1-yl] acetohydrazide $6 b$

IR (KBr, cm-1): 3163 (N-H sec.), 3047 (C-H str. ArH), 1699 (C=0), 1541 and $1440(\mathrm{C}=\mathrm{C}), 1274(\mathrm{C}-\mathrm{N}){ }^{1}{ }^{1} \mathrm{H}$ NMR (DMSO- $\left.d_{6}, 400 \mathrm{MHz}\right) \delta: 4.73$ (s, $2 \mathrm{H}, \mathrm{CH}_{2}$ ), 7.13-7.17 (m, 2H, benzimidazole), 7.36-7.44 (m, $4 \mathrm{H}$, phenyl), 7.51-7.56 (m, 4H, phenyl), 8.11-8.13 (d, 2H, benzimidazole), $8.72(\mathrm{~s}, 1 \mathrm{H}$, $\mathrm{N}=\mathrm{CH}), 10.97$ (s, 1H, OH), 11.23 (1H, CONH); ES-MS (m/z): 371 [M+1]; Anal. calcd for $\mathrm{C}_{22} \mathrm{H}_{18} \mathrm{~N}_{4} \mathrm{O}_{2}$ : C, 71.34; $\mathrm{H}, 4.90 ; \mathrm{N}, 15.13$. Found: C, 71.46; $\mathrm{H}, 4.82 ; \mathrm{N}, 15.02$.

$N$-(2-Chlorobenzylidene)-2-[2-(phenyl)-1H-benzimidazol-1-yl) acetohydrazide $6 c$

IR (KBr, cm ${ }^{-1}$ ): 3159 (N-H sec.), 3047 (C-H str. ArH), 1653 (C=0), 1626 and 1464 (C=C), 1276 (C-N), 742 (C-Cl); ${ }^{1} \mathrm{H}$ NMR (DMSO-d $d_{6}, 400 \mathrm{MHz}$ ) $\delta: 4.69\left(\mathrm{~s}, 2 \mathrm{H}, \mathrm{CH}_{2}\right), 7.13-7.18(\mathrm{~m}, 2 \mathrm{H}$, benzimidazole), 7.37-7.46 (m, 
3H, phenyl), 7.53-7.55 (m, 2H, phenyl), 7.84-7.85 (m, 4H, phenyl), 8.11$8.13(\mathrm{~d}, 2 \mathrm{H}$, benzimidazole), $8.63(\mathrm{~s}, 1 \mathrm{H}, \mathrm{N}=\mathrm{CH}), 11.37(1 \mathrm{H}, \mathrm{CONH})$; ESMS (m/z): 389 [M+1]; Anal. calcd for $\mathrm{C}_{22} \mathrm{H}_{17} \mathrm{~N}_{4} \mathrm{OCl}$ : C, 67.95; $\mathrm{H}, 4.41 ; \mathrm{N}$, 14.41. Found: C, 67.86; H, 4.46; N, 14.42 .

\section{$N$-Benzylidene-2-\{2-(2-hydroxyphenyl)-1H-benzimidazol-1-yl\} acetohydrazide $6 d$}

IR (KBr, cm ${ }^{-1}$ ): 3234 (O-H), 3061 (C-H str. ArH), 2916 (C-H str. aliphatic), $1651(\mathrm{C}=0), 1629$ and 1489 (C=C), 1273 (C-N), 738 (ortho subst. oop); ${ }^{1} \mathrm{H}$ NMR (DMSO- $\left.d_{6}, 400 \mathrm{MHz}\right) \delta: 4.76\left(\mathrm{~s}, 2 \mathrm{H}, \mathrm{CH}_{2}\right), 6.88-6.97(\mathrm{~m}, 2 \mathrm{H}$, benzimidazole), 7.19-7.23 (m, 3H, phenyl), 7.26-7.27 (d, 1H, phenyl), 7.38-7.40 (m, 3H, phenyl), 7.52-7.56 (m, 2H, benzimidazole), 7.62-7.63 (m, 2H, phenyl), 7.97-7.99 (s, 1H, OH), $8.69(\mathrm{~s}, 1 \mathrm{H}, \mathrm{N}=\mathrm{CH}), 11.27(1 \mathrm{H}$, CONH); ES-MS (m/z): 371 [M+1]; Anal. calcd for $\mathrm{C}_{22} \mathrm{H}_{18} \mathrm{~N}_{4} \mathrm{O}_{2}: \mathrm{C}, 71.34 ; \mathrm{H}$, 4.90; N, 15.13. Found: C, 71.38; H, 4.91; N, 15.01

N-(2-Hydroxybenzylidene)-2-\{2-(2-hydroxyphenyl)-1Hbenzimidazol-1-yl\} acetohydrazide $6 e$

IR ( $\mathrm{KBr}, \mathrm{cm}^{-1}$ ): 3238 (O-H), 3059 (C-H str. ArH), 2918 (C-H str. aliphatic), 1629 (C=0), 1591 and 1489 (C=C), 1259 (C-N), 738 (ortho subst. oop); ${ }^{1} \mathrm{H}$ NMR (DMSO- $\left.d, 400 \mathrm{MHz}\right) \delta: 4.84\left(\mathrm{~s}, 2 \mathrm{H}, \mathrm{CH}_{2}\right), 6.89-6.96(\mathrm{~m}, 2 \mathrm{H}$, benzimidazole), 7.18-7.23 (m, 3H, phenyl), 7.26-7.27 (d, 1H, phenyl), 7.38-7.44 (m, 4H, phenyl), 7.54-7.56 (m, 2H, benzimidazole), 7.95-7.98 $(\mathrm{s}, 2 \mathrm{H}, \mathrm{OH}), 8.54(\mathrm{~s}, 1 \mathrm{H}, \mathrm{N}=\mathrm{CH}), 11.03(1 \mathrm{H}, \mathrm{CONH}) ; \mathrm{ES}-\mathrm{MS}(\mathrm{m} / \mathrm{z}): 387$ $[\mathrm{M}+1]$; Anal. calcd for $\mathrm{C}_{22} \mathrm{H}_{18} \mathrm{~N}_{4} \mathrm{O}_{3}: \mathrm{C}, 68.38 ; \mathrm{H}, 4.70 ; \mathrm{N}, 14.50$. Found: $\mathrm{C}$, 68.42; H, 4.62; N, 14.42

$N$-(2-Chlorobenzylidene)-2-\{2-(2-hydroxyphenyl)-1Hbenzimidazol-1-yl\}acetohydrazide $6 f$

IR ( $\mathrm{KBr}, \mathrm{cm}^{-1}$ ): 3238 (O-H), 3061 (C-H str. ArH), 2918 (C-H str. aliphatic), $1629(\mathrm{C}=0), 1591 \& 1489$ (C=C), 1259 (C-N), 738 (ortho subst. oop); ${ }^{1} \mathrm{H}$ NMR (DMSO- $\left.d, 400 \mathrm{MHz}\right) \delta: 4.62\left(\mathrm{~s}, 2 \mathrm{H}, \mathrm{CH}_{2}\right), 6.89-6.96(\mathrm{~m}, 2 \mathrm{H}$, benzimidazole), 7.19-7.22 (m, 3H, phenyl), 7.26-7.30 (d, 1H, phenyl), 7.32-7.41 (m, 4H, phenyl), 7.56-7.58 (m, 2H, benzimidazole), 7.95-7.98 (s, 1H, OH), $8.21(\mathrm{~s}, 1 \mathrm{H}, \mathrm{N}=\mathrm{CH}), 10.98(1 \mathrm{H}, \mathrm{CONH})$; ES-MS $(\mathrm{m} / \mathrm{z}): 405$ $[\mathrm{M}+1]$; Anal. calcd for $\mathrm{C}_{22} \mathrm{H}_{17} \mathrm{~N}_{4} \mathrm{O}_{2} \mathrm{Cl}: \mathrm{C}, 65.27 ; \mathrm{H}, 4.23 ; \mathrm{N}, 13.84$. Found: C, 65.22; H, 4.19; N, 13.62 .

$N$-Benzylidene-2-\{2-(2-chlorophenyl)-1H-benzimidazol-1-yl\} acetohydrazide $6 g$

IR ( $\mathrm{KBr}, \mathrm{cm}^{-1}$ ): 3242 ( $\mathrm{N}-\mathrm{H}$ sec.), 3061 (C-H str. ArH), 2916 (C-H str. aliphatic), $1651(\mathrm{C}=0), 1626 \& 1491(\mathrm{C}=\mathrm{C}), 1273(\mathrm{C}-\mathrm{N}), 746(\mathrm{C}-\mathrm{Cl})$; ${ }^{1} \mathrm{H}$ NMR (DMSO- $\left.d_{6}, 400 \mathrm{MHz}\right) \delta: 4.32\left(\mathrm{~s}, 2 \mathrm{H}, \mathrm{CH}_{2}\right), 7.17-7.19(\mathrm{~m}, 2 \mathrm{H}$, benzimidazole), 7.37-7.44 (m, 6H, phenyl), 7.49-7.51 (d, 1H, phenyl), 7.58-7.60 (m, 2H, benzimidazole), 7.87-7.90 (d, 2H, phenyl), 8.12 (s, $1 \mathrm{H}, \mathrm{N}=\mathrm{CH}), 10.84(1 \mathrm{H}, \mathrm{CONH}) ; \mathrm{ES}-\mathrm{MS}(\mathrm{m} / \mathrm{z}): 389[\mathrm{M}+1]$; Anal. calcd for $\mathrm{C}_{22} \mathrm{H}_{17} \mathrm{~N}_{4} \mathrm{OCl}$ : C, 67.95; $\mathrm{H}, 4.41 ; \mathrm{N}, 14.41$. Found: $\mathrm{C}, 67.79 ; \mathrm{H}, 4.37 ; \mathrm{N}$, 14.42

$N$-(2-Hydroxybenzylidene)-2-\{2-(2-chlorophenyl)-1Hbenzimidazol-1-yl\}acetohydrazide $6 \mathrm{~h}$

IR (KBr, cm$\left.{ }^{-1}\right)$ : 3254 (O-H), 3061 (C-H str. ArH), 2916 (C-H str. aliphatic), 1651 (C=0), $1624 \& 1489$ (C=C), 1274 (C-N), 742 (C-Cl); ${ }^{1} \mathrm{H}$ NMR (DMSO- $\left.d_{6}, 400 \mathrm{MHz}\right) \delta: 4.52\left(\mathrm{~s}, 2 \mathrm{H}, \mathrm{CH}_{2}\right), 7.16-7.20(\mathrm{~m}, 2 \mathrm{H}$, benzimidazole), 7.36-7.43 (m, 7H, phenyl), 7.47-7.51 (d, 1H, phenyl), 7.57-7.61 (m, 2H, benzimidazole), 7.88-7.90 (s, $1 \mathrm{H}, \mathrm{OH}), 8.32(\mathrm{~s}, 1 \mathrm{H}$, $\mathrm{N}=\mathrm{CH}), 11.15(1 \mathrm{H}, \mathrm{CONH})$; ES-MS $(\mathrm{m} / \mathrm{z}): 405[\mathrm{M}+1]$; Anal. calcd for $\mathrm{C}_{22} \mathrm{H}_{17} \mathrm{~N}_{4} \mathrm{O}_{2} \mathrm{Cl}$ : C, 65.27; $\mathrm{H}, 4.23 ; \mathrm{N}, 13.84$. Found: $\mathrm{C}, 65.12 ; \mathrm{H}, 4.34 ; \mathrm{N}$, 13.71 .

$N$-(2-Chlorobenzylidene)-2-\{2-(2-chlorophenyl)-1Hbenzimidazole-1-yl\}acetohydrazide $6 i$

IR (KBr, cm ${ }^{-1}$ ): 3157 (N-H sec.), 3061 (C-H str. ArH), 2918 (C-H str. aliphatic), $1651(\mathrm{C}=0), 1622$ and 1442 (C=C), 1273 (C-N), 746 (C$\mathrm{Cl}) ;{ }^{1} \mathrm{H}$ NMR $\left(\mathrm{CDCl}_{3}, 400 \mathrm{MHz}\right) \delta: 4.56\left(\mathrm{~s}, 2 \mathrm{H}, \mathrm{CH}_{2}\right), 7.32-7.35(\mathrm{~m}$, $2 \mathrm{H}$, benzimidazole), 7.42-7.45 (m, 7H, phenyl), 7.57-7.61 $(\mathrm{m}, 2 \mathrm{H}$, benzimidazole), 7.70-7.71 (d, $1 \mathrm{H}$, phenyl), 8.45-8.50 (s, $1 \mathrm{H}, \mathrm{N}=\mathrm{CH})$, 11.27 (1H, CONH); ES-MS ( $\mathrm{m} / \mathrm{z}): 424$ [M+1]; Anal. calcd for $\mathrm{C}_{22} \mathrm{H}_{16} \mathrm{~N}_{4} \mathrm{OCl}$ : C, 62.42; H, 3.81; N, 13.24. Found: C, 62.38; H, 3.91; N, 13.36 .

N-Benzylidene-2-\{2-(3-chlorophenyl)-1H-benzimidazol-1-yl\} acetohydrazide $6 j$

IR ( $\mathrm{KBr}, \mathrm{cm}^{-1}$ ): 3159 (N-H sec.), 3043 (C-H str. ArH), 2918 (C-H str. aliphatic), $1651(\mathrm{C}=0), 1591$ \& $1469(\mathrm{C}=\mathrm{C}), 1284$ (C-N), 744 (C$\mathrm{Cl}$ ); ${ }^{1} \mathrm{H}$ NMR (DMSO- $\left.d, 400 \mathrm{MHz}\right) \delta: 4.51\left(\mathrm{~s}, 2 \mathrm{H}, \mathrm{CH}_{2}\right), 7.15-7.19(\mathrm{~m}$, $2 \mathrm{H}$, benzimidazole), 7.40-7.49 (m, 8H, phenyl), 7.54-7.57 (m, $2 \mathrm{H}$, benzimidazole), 8.08-8.10 (d, $1 \mathrm{H}$, phenyl), 8.19-8.20 (s, $1 \mathrm{H}, \mathrm{N}=\mathrm{CH}$ ), $10.98(1 \mathrm{H}, \mathrm{CONH})$; ES-MS $(\mathrm{m} / \mathrm{z}): 389$ [M+1]; Anal. calcd for $\mathrm{C}_{22} \mathrm{H}_{17} \mathrm{~N}_{4} \mathrm{OCl}$ : C, 67.95; H, 4.41; N, 14.41. Found: C, 67.84; H, 4.46; N, 14.44.

$N$-(2-Hydroxybenzylidene-2-\{2-(3-chlorophenyl)-1Hbenzimidazol-1-yl\}acetohydrazide $6 k$

IR (KBr, cm-1): 3161 (O-H), 3043 (C-H str. ArH), 2918 (C-H str. aliphatic), $1622(\mathrm{C}=0), 1572$ and $1467(\mathrm{C}=\mathrm{C}), 1228(\mathrm{C}-\mathrm{N}), 744$ (C$\mathrm{Cl}$ ); ${ }^{1} \mathrm{H}$ NMR (DMSO- $\left.d_{6}, 400 \mathrm{MHz}\right) \delta: 4.59\left(\mathrm{~s}, 2 \mathrm{H}, \mathrm{CH}_{2}\right), 7.14-7.19(\mathrm{~m}$, $2 \mathrm{H}$, benzimidazole), 7.38-7.47 (m, 7H, phenyl), 7.53-7.56 (m, $2 \mathrm{H}$, benzimidazole), 8.07-8.09 (d, $1 \mathrm{H}$, phenyl), 8.19-8.20 (s, $1 \mathrm{H}, \mathrm{N}=\mathrm{CH})$, $11.02(1 \mathrm{H}, \mathrm{CONH})$; ES-MS (m/z): 405 [M+1]; Anal. calcd for $\mathrm{C}_{22} \mathrm{H}_{17} \mathrm{~N}_{4} \mathrm{O}_{2} \mathrm{Cl}$ : C, 65.27; H, 4.23; N, 13.84. Found: C, 65.52; H, 4.12; N, 13.76.

$N$-(2-Chlorobenzylidene-2-\{2-(3-chlorophenyl) - 1 Hbenzimidazol-1-yl\}acetohydrazide 61

IR ( $\left.\mathrm{KBr}, \mathrm{cm}^{-1}\right)$ : 3159 (N-H), 3043 (C-H str. ArH), 2964 (C-H str. aliphatic), $1651(\mathrm{C}=0), 1572$ \& $1440(\mathrm{C}=\mathrm{C}), 1359(\mathrm{C}-\mathrm{N}), 744(\mathrm{C}-\mathrm{Cl}), 895$ and 680 (meta oop); ${ }^{1} \mathrm{H}$ NMR (DMSO- $\left.d_{6}, 400 \mathrm{MHz}\right) \delta: 4.74\left(\mathrm{~s}, 2 \mathrm{H}, \mathrm{CH}_{2}\right.$ ), 7.16-7.20 (m, 2H, benzimidazole), 7.42-7.51 (m, 7H, phenyl), 7.55-7.57 $(\mathrm{m}, 2 \mathrm{H}$, benzimidazole), 8.08-8.11 (d, $1 \mathrm{H}$, phenyl), $8.20(\mathrm{~s}, 1 \mathrm{H}, \mathrm{N}=\mathrm{CH}), 11.12(1 \mathrm{H}$, CONH); ES-MS (m/z): 424 [M+1]; Anal. calcd for $\mathrm{C}_{22} \mathrm{H}_{16} \mathrm{~N}_{4} \mathrm{OCl}_{2}$ : C, 62.42; H, 3.81; N, 13.24. Found: C, 62.19; H, 3.83; N, 13.12 .

The physiochemical characterization of synthesized derivatives is presented in Table 1.

\section{Antimicrobial activity}

All the synthesized benzimidazole-hydrazone derivatives $6 a-61$ were screened for their in vitro antibacterial activity against Staphylococcus aureus, Bacillus subtilis, Escherichia coli, and Pseudomonas aeruginosa using agar diffusion method (cup plate method). Ciprofloxacin was used as standard drugs for antibacterial activity. Three different concentrations $(25,50$ and $100 \mu \mathrm{g} / \mathrm{ml})$ of synthesized derivatives were used to evaluate antimicrobial potential, and the results have been summarized in Table 2.

\section{RESULTS AND DISCUSSION}

2-(Substituted phenyl)-1H-benzimidazole $3 a-d$ was synthesized by refluxing $o$-phenylenediamine 1 with appropriately substituted benzoic acid $2 \mathrm{a}-\mathrm{d}$ in the presence of polyphosphoric acid. The compounds 3a-d on further reaction with ethylchloroacetate in the presence of base and dry acetone gave ethyl-2-[2-(substituted phenyl)-1H-benzimidazol1-yl]acetate $4 \mathrm{a}-\mathrm{d}$. In the next step, substituted esters reacted with hydrazine hydrate to form 2-[2-(substituted phenyl)- $1 \mathrm{H}$ - benzimidazol1-yl]acetohydrazide 5a-d. Finally, benzimidazole-hydrazone derivatives $6 \mathrm{a}-\mathrm{l}$ were synthesized by treatment of $5 \mathrm{a}-\mathrm{d}$ with substituted aldehydes in the presence of ethanol and little acetic acid.

Formulae of the synthesized compounds were confirmed by elemental analysis, and their chemical structures were elucidated by IR, ${ }^{1} \mathrm{H}$ NMR and electrospray mass spectrometry spectra. In the IR spectra, the vibrational bands due to $\mathrm{N}-\mathrm{H}, \mathrm{C}=\mathrm{O}, \mathrm{C}=\mathrm{C}$ and $\mathrm{C}=\mathrm{N}$ vibrations appeared in the expected regions. The formation of benzimidazole nucleus was confirmed by the appearance of single band of sec. N-H str. vibrations at $3045-3404 \mathrm{~cm}^{-1}$. The characteristic $\mathrm{C}=0$ str. vibrations at $1735-1749$ and 1610-1620 confirmed the formation of ester $4 \mathrm{a}-4 \mathrm{~d}$ and amide $5 \mathrm{a}-$ 
Table 1: Physiochemical characteristics 3a-d, 4a-d, 5a-d and 6a-d

\begin{tabular}{|c|c|c|c|c|c|c|c|}
\hline Compound number & $\mathbf{R}$ & $\mathbf{R}^{1}$ & Molecular formula & Molecular weight & M.p $\left({ }^{\circ} \mathrm{C}\right)$ & $\mathbf{R}_{\mathrm{f}}$ value $^{\mathrm{a}}$ & Yield (\%) \\
\hline $3 a$ & $-\mathrm{H}$ & - & $\mathrm{C}_{13} \mathrm{H}_{10} \mathrm{~N}_{2}$ & 194.23 & $294-296$ & 0.73 & 82.63 \\
\hline $3 b$ & $2-\mathrm{OH}$ & - & $\mathrm{C}_{13}^{13} \mathrm{H}_{10} \mathrm{~N}_{2}^{2} \mathrm{O}$ & 210.23 & $239-241$ & 0.67 & 69.34 \\
\hline $3 c$ & $2-\mathrm{Cl}$ & - & $\mathrm{C}_{13}^{13} \mathrm{H}_{9} \mathrm{~N}_{2} \mathrm{Cl}$ & 228.67 & $230-231$ & 0.71 & 72.12 \\
\hline $3 d$ & $3-\mathrm{Cl}$ & - & $\mathrm{C}_{13}^{13} \mathrm{H}_{9} \mathrm{~N}_{2} \mathrm{Cl}^{2}$ & 228.67 & $232-234$ & 0.69 & 68.42 \\
\hline $4 a$ & $-\mathrm{H}$ & - & $\mathrm{C}_{17} \mathrm{H}_{16} \mathrm{~N}_{2} \mathrm{O}_{2}$ & 280.32 & $253-255$ & 0.71 & 58.21 \\
\hline $4 b$ & $2-\mathrm{OH}$ & - & $\mathrm{C}_{17}^{17} \mathrm{H}_{16} \mathrm{~N}_{2}^{2} \mathrm{O}_{3}^{2}$ & 296.32 & $253-255$ & 0.74 & 62.47 \\
\hline $4 c$ & $2-\mathrm{Cl}$ & - & $\mathrm{C}_{17} \mathrm{H}_{15} \mathrm{~N}_{2} \mathrm{O}_{2} \mathrm{Cl}$ & 314.76 & $249-251$ & 0.76 & 77.26 \\
\hline $5 a$ & $-\mathrm{H}$ & - & $\mathrm{C}_{15} \mathrm{H}_{14} \mathrm{~N}_{4} \mathrm{O}$ & 266.29 & $262-264$ & 0.71 & 65.58 \\
\hline $5 b$ & $2-\mathrm{OH}$ & - & $\mathrm{C}_{15}^{15} \mathrm{H}_{14}^{14} \mathrm{~N}_{4}^{4} \mathrm{O}_{2}$ & 282.29 & $268-280$ & 0.76 & 69.23 \\
\hline $5 c$ & $2-\mathrm{Cl}$ & - & $\mathrm{C}_{15} \mathrm{H}_{13} \mathrm{~N}_{4} \mathrm{OCl}$ & 300.74 & $273-275$ & 0.74 & 73.27 \\
\hline $5 d$ & $3-\mathrm{Cl}$ & - & $\mathrm{C}_{15} \mathrm{H}_{13} \mathrm{~N}_{4} \mathrm{OCl}$ & 300.74 & $267-269$ & 0.72 & 68.27 \\
\hline $6 a$ & $-\mathrm{H}$ & $-\mathrm{H}$ & $\mathrm{C}_{22} \mathrm{H}_{18} \mathrm{~N}_{4} \mathrm{O}$ & 354.40 & $277-279$ & 0.85 & 74.81 \\
\hline $6 b$ & $-\mathrm{H}$ & $2-\mathrm{OH}$ & $\mathrm{C}_{22}^{22} \mathrm{H}_{18} \mathrm{~N}_{4} \mathrm{O}_{2}$ & 370.40 & $276-278$ & 0.83 & 75.61 \\
\hline $6 c$ & $-\mathrm{H}$ & $2-\mathrm{Cl}$ & $\mathrm{C}_{22} \mathrm{H}_{17} \mathrm{~N}_{4} \mathrm{OCl}$ & 388.84 & $279-281$ & 0.79 & 77.61 \\
\hline $6 d$ & $2-\mathrm{OH}$ & $-\mathrm{H}$ & $\mathrm{C}_{22} \mathrm{H}_{18} \mathrm{~N}_{4}^{4} \mathrm{O}_{2}$ & 370.40 & $271-273$ & 0.77 & 78.56 \\
\hline $6 g$ & $2-\mathrm{Cl}$ & $-\mathrm{H}$ & $\mathrm{C}_{22}^{22} \mathrm{H}_{17} \mathrm{~N}_{4}^{4} \mathrm{OCl}$ & 388.84 & $283-285$ & 0.74 & 73.86 \\
\hline $6 \mathrm{~h}$ & $2-\mathrm{Cl}$ & $2-\mathrm{OH}$ & $\mathrm{C}_{22}^{22} \mathrm{H}_{17} \mathrm{~N}_{4} \mathrm{O}_{2} \mathrm{Cl}$ & 404.84 & $268-271$ & 0.86 & 62.94 \\
\hline $6 i$ & $2-\mathrm{Cl}$ & $2-\mathrm{Cl}$ & $\mathrm{C}_{22}^{22} \mathrm{H}_{16} \mathrm{~N}_{4}^{4} \mathrm{OCl}_{2}$ & 423.29 & $277-279$ & 0.76 & 78.96 \\
\hline $6 j$ & $3-\mathrm{Cl}$ & $-\mathrm{H}$ & $\mathrm{C}_{22}^{22} \mathrm{H}_{17} \mathrm{~N}_{4} \mathrm{OCl}^{2}$ & 388.84 & $272-274$ & 0.79 & 57.27 \\
\hline $6 \mathrm{k}$ & $3-\mathrm{Cl}$ & $2-\mathrm{OH}$ & $\mathrm{C}_{22}^{22} \mathrm{H}_{17} \mathrm{~N}_{4}^{4} \mathrm{O}_{2} \mathrm{Cl}$ & 404.84 & $277-279$ & 0.76 & 59.67 \\
\hline 61 & $3-\mathrm{Cl}$ & $2-\mathrm{Cl}$ & $\mathrm{C}_{22}^{22} \mathrm{H}_{16}^{17} \mathrm{~N}_{4}^{4} \mathrm{OCl}_{2}^{2}$ & 423.29 & $279-281$ & 0.81 & 64.57 \\
\hline
\end{tabular}

aTLC mobile phase:hexane:ethyl acetate:methanol (6:3.5:0.5)

Table 2: Antibacterial evaluation of benzimidazole-hydrazone derivatives 6a-1

\begin{tabular}{|c|c|c|c|c|c|c|c|c|c|c|c|c|}
\hline \multirow{2}{*}{$\begin{array}{l}\text { Bacterial strain } \\
\text { Conc. }(\mu \mathrm{g} / \mathrm{ml})\end{array}$} & \multicolumn{3}{|c|}{ S. aureus } & \multicolumn{3}{|c|}{ B. subtilis } & \multicolumn{3}{|c|}{ E. coli } & \multicolumn{3}{|c|}{ P. aeruginosa } \\
\hline & 25 & 50 & 100 & 25 & 50 & 100 & 25 & 50 & 100 & 25 & 50 & 100 \\
\hline Compound number & \multicolumn{12}{|c|}{ Zone of inhibition (mm) } \\
\hline $6 a$ & 20 & 29 & 31 & 13 & 17 & 21 & 19 & 23 & 26 & 21 & 28 & 31 \\
\hline $6 b$ & 23 & 26 & 27 & 20 & 26 & 28 & 17 & 26 & 32 & 17 & 23 & 27 \\
\hline $6 c$ & 22 & 28 & 31 & 14 & 19 & 21 & 21 & 26 & 29 & 17 & 26 & 32 \\
\hline $6 \mathrm{~d}$ & 20 & 27 & 32 & 19 & 28 & 29 & 22 & 27 & 31 & 19 & 24 & 26 \\
\hline $6 e$ & 21 & 29 & 34 & 16 & 23 & 27 & 23 & 29 & 33 & 25 & 29 & 31 \\
\hline $6 f$ & 25 & 32 & 37 & 17 & 29 & 31 & 25 & 32 & 34 & 26 & 32 & 33 \\
\hline $6 \mathrm{~g}$ & 15 & 19 & 21 & 11 & 17 & 19 & 15 & 21 & 25 & 17 & 21 & 23 \\
\hline $6 \mathrm{~h}$ & 16 & 18 & 22 & 13 & 18 & 22 & 12 & 15 & 19 & 15 & 19 & 20 \\
\hline $6 i$ & 15 & 19 & 22 & 15 & 19 & 23 & 13 & 18 & 22 & 18 & 22 & 23 \\
\hline $6 j$ & 11 & 16 & 21 & 16 & 19 & 21 & 14 & 17 & 21 & 16 & 20 & 22 \\
\hline $6 \mathrm{k}$ & 15 & 20 & 23 & 19 & 22 & 24 & 11 & 14 & 18 & 15 & 19 & 23 \\
\hline 61 & 15 & 19 & 23 & 16 & 18 & 21 & 10 & 15 & 17 & 16 & 20 & 25 \\
\hline Ciprofloxacin & 19 & 21 & 23 & 23 & 27 & 29 & 23 & 25 & 29 & 23 & 25 & 27 \\
\hline
\end{tabular}

S. aureus: Staphylococcus aureus, B. subtilis: Bacillus subtilis, E. coli: Escherichia coli, P. aeruginosa: Pseudomonas aeruginosa

$5 \mathrm{~d}$ derivatives, respectively. The out-of-plane bending vibrations that appeared in a range of 650-750 were used to assign substitution on aromatic ring. The ${ }^{1} \mathrm{H}$ NMR spectra showed amino proton of secondary amine at 12.73-12.84 ppm in compounds $3 \mathrm{a}-3 \mathrm{~d}$ which confirmed the formation of benzimidazole nucleus. The aromatic protons of phenyl ring at $2^{\text {nd }}$ position of benzimidazole nucleus appeared at 7.1-8.1 ppm in all the derivatives with variable multiplicity. The protons of ethyl chain attached to ester functional group in $4 \mathrm{a}-4 \mathrm{~d}$ were observed at around 1.18 and $4.2 \mathrm{ppm}$ respectively. The formation of hydrazides $5 a-5 d$ and benzimidazole-hydrazone derivatives $6 a-61$ was confirmed by appearance of $\mathrm{NH}$ protons in expected regions. Elemental analysis results were found to be satisfactory in all the compounds. In mass spectra, $M+1$ peaks in all the compounds were in agreement with their molecular formula.

The synthesized titled compounds exhibited moderate to strong antibacterial activity against both Gram-positive and Gram-negative bacteria. The compound having 2-hydroxyphenyl ring at $2^{\text {nd }}$ position and 2-chloro substituent on phenyl ring at $1^{\text {st }}$ position of benzimidazole nucleus $6 \mathrm{f}$ was found to be most active against all the stains of bacteria exhibiting zone of inhibition of $37,31,34$, and $33 \mathrm{~mm}$ at concentration of $100 \mu \mathrm{g} / \mathrm{ml}$ against S. aureus, B. subtilis, E. coli, and P. aeruginosa, respectively. The chloro substituent at ortho and meta positions of phenyl ring attached to $2^{\text {nd }}$ position of benzimidazole nucleus decreased the antibacterial potency of molecules against both Gram-positive and Gram-negative bacteria in dose-dependent manner. The compounds 6a-6f have emerged as the most effective antimicrobial agents against S. aureus, E. coli, and P. aeruginosa, whereas compounds $6 \mathrm{~b}$ was the most active against $B$. subtilis.

\section{CONCLUSION}

A novel series of $N$-(substituted benzylidene)-2-[(2-(substituted phenyl)-1H-benzimidazol-1-yl) acetohydrazide derivatives 6a-l had been synthesized and characterized by IR, NMR, mass and elemental analysis. The final compounds were screened for in vitro antibacterial activity against both Gram-positive and Gram-negative strains of bacteria by cup-plate method. Among the various derivative, the compounds 6a-6f excellent inhibition of bacterial growth as compared to standard drug ciprofloxacin. 


\section{ACKNOWLEDGMENT}

The authors are thankful to Panjab University, Chandigarh, for providing spectral and analytical data of synthesized compounds.

\section{REFERENCES}

1. Ozkay Y, Tunali Y, Karaca H, Isikdag I. Antimicrobial activity and a SAR study of some novel benzimidazole derivatives bearing hydrazone moiety. Eur J Med Chem 2010;45(8):3293-8.

2. Rai J, Randhawa GK, Kaur M. Recent advances in antibacterial drugs. Int J Appl Basic Med Res 2013;3:3-10.

3. Sharma A. Antimicrobial resistance: No action today, no cure tomorrow. Indian J Med Microbiol 2011;29:91-2.

4. While A. No action today means no cure tomorrow: The threat of antimicrobial resistance. Br J Community Nurs 2016;21(7):344-7.

5. Santhanamari TH, Meenakshi PR, Velayutham SR. In vitro antibacterial activity of extracts of Lawsonia inermis and Punica grantum against clinically isolated antibiotic resistant Pseudomonas aeruginosa and Staphylococcus aureus. Asian J Pharm Clin Res 2011;4:62-5.

6. Zhang D, Wang Z, Xu W, Sun F, Tang L, Wang J. Design, synthesis and antibacterial activity of novel actinonin derivatives containing benzimidazole heterocycles. Eur J Med Chem 2009;44(5):2202-10.

7. Marinescu M, Tudorache DG, Marton GI, Zalaru CM, Popa M, Chifiriuc MC, et al. Density functional theory molecular modeling, chemical synthesis, and antimicrobial behavior of selected benzimidazole derivatives. J Mol Struct 2017;1130:463-71.

8. Sharma D, Narasimhan B, Kumar P, Jalbout A. Synthesis and QSAR evaluation of 2-(substituted phenyl)-1H-benzimidazoles and [2-(substituted phenyl)-benzimidazol-1-yl]-pyridin-3-yl-methanones. Eur J Med Chem 2009;44:1119-27.

9. Apohan E, Yilmaz U, Yilmaz O, Serindag A, Küçükbay H, Yesilada O, et al. Synthesis, cytotoxic and antimicrobial activities of novel cobalt and zinc complexes of benzimidazole derivatives. J Organomet Chem 2017;828:52-8.

10. Zhu W, Da Y, Wu D, Zheng H, Zhu L, Wang L, et al. Design, synthesis and biological evaluation of new 5-nitro benzimidazole derivatives as AT 1 antagonists with anti-hypertension activities. Bioorg Med Chem 2014;22:2294-302

11. Bansal Y, Silakari O. The therapeutic journey of benzimidazoles: A review. Bioorg Med Chem 2012;20(21):6208-36.

12. Lavanya P, Suresh M, Kotaiah Y, Harikrishna N, Rao CV. Synthesis, antibacterial, antifungal and antioxidant activity studies on 6-bromo2-substitutedphenyl-1H-imidazo [4,5-b] pyridine. Asian J Pharm Clin Res 2011;4:69-73.

13. Tonelli M, Novelli F, Tasso B, Vazzana I, Sparatore A, Boido V, et al. Antiviral activity of benzimidazole derivatives. III. Novel anti-CVB-5, anti-RSV and anti-Sb-1 agents. Bioorg Med Chem 2014;22:4893-909.

14. Gaba M, Gaba P, Uppal D, Dhingra N, Bahia MS, Silakari O, et al. Benzimidazole derivatives: Search for GI-friendly anti-inflammatory analgesic agents. Acta Pharm Sin B 2015;5:337-42.

15. Achar KC, Hosamani KM, Seetharamareddy HR. In-vivo analgesic and anti-inflammatory activities of newly synthesized benzimidazole derivatives. Eur J Med Chem 2010;45(5):2048-54.

16. Roopashree R, Mohan CD, Swaroop TR, Jagadish S, Rangappa KS. Synthesis, characterization, and in vivo biological evaluation of novel benzimidazoles as potential anticancer agents. Asian J Pharm Clin Res 2014;7:309-13.

17. Da Settimo A, Primofiore G, Da Settimo F, Calzolari L, Cazzulani P, Passoni A, et al. 1-Substituted 2-benzylaminobenzimidazole derivatives: Compounds with H1-antihistaminic activity. Eur J Med Chem 1992;27:395-400.

18. Cereda E, Turconi M, Ezhaya A, Bellora E, Brambilla A, Pagani F, et al. Anti-secretory and anti-ulcer activities of some new 2-(2-pyridylmethylsulfinyl)-benzimidazoles. Eur J Med Chem 1987;22:527-37.

19. Tunçbilek M, Kiper T, Altanlar N. Synthesis and in vitro antimicrobial activity of some novel substituted benzimidazole derivatives having potent activity against MRSA. Eur J Med Chem 2009;44(3):1024-33.

20. Bui HT, Ha QT, Oh WK, Vo DD, Chau YN, Tu CT, et al. Microwave assisted synthesis and cytotoxic activity evaluations of new benzimidazole derivatives. Tetrahedron Lett 2016;57:887-91.

21. Wen J, Luo YL, Zhang HZ, Zhao HH, Zhou CH, Cai GX. A green and convenient approach toward benzimidazole derivatives and their antimicrobial activity. Chin Chem Lett 2016;27:391-4.

22. Mehranpour AM, Zahiri M. Synthesis and characterization of new benzimidazole derivatives using 2-substituted 1,3-bis(dimethylamino)trimethinium salts. Tetrahedron Lett 2014;55:3969-71.

23. Fang XJ, Jeyakkumar P, Avula SR, Zhou Q, Zhou CH. Design, synthesis and biological evaluation of 5-fluorouracil-derived benzimidazoles as novel type of potential antimicrobial agents. Bioorg Med Chem Lett 2016;26(11):2584-8.

24. Asif M, Husain A. Analgesic, anti-Inflammatory, and antiplatelet profile of hydrazones containing synthetic molecules. J Appl Chem 2013. DOI: $10.1155 / 2013 / 247203$.

25. Popiolek L. Hydrazide-hydrazones as potential antimicrobial agents: Overview of the literature since 2010. Med Chem Res 2017;26(2):287-301.

26. Soltani Rad MN, Khalafi-Nezhad A, Behrouz S. Synthesis of some novel hydrazono acyclic nucleoside analogues. Beilstein J Org Chem 2010;6:49.

27. Malik MA, Al-Thabaiti SA, Malik MA. Synthesis, structure optimization and antifungal screening of novel tetrazole ring bearing acyl-hydrazones. Int J Mol Sci 2012;13:10880-98.

28. Özkay Y, Tunali Y, Karaca H, Isikdag I. Antimicrobial activity of a new series of benzimidazole derivatives. Arch Pharm Res 2011;34(9):1427-35

29. Hein DW, Alheim RJ, Leavitt JJ. The use of polyphosphoric acid in the synthesis of 2-aryl-and 2-alkyl-substituted benzimidazoles, benzoxazoles and benzothiazoles1. J Am Chem Soc 1957;79:427-9.

30. Paul K, Bindal S, Luxami V. Synthesis of new conjugated coumarinbenzimidazole hybrids and their anticancer activity. Bioorg Med Chem Lett 2013;23(12):3667-72.

31. Ansari KF, Lal C. Synthesis and evaluation of some new benzimidazole derivatives as potential antimicrobial agents. Eur J Med Chem 2009;44(5):2294-9.

32. Shaharyar M, Mazumder A, Ahsan MJ Synthesis, characterization and anticancer evaluation of 2-(naphthalen-1-ylmethyl/naphthalen2-yloxymethyl)-1-[5-(substituted phenyl)-[1, 3, 4] oxadiazol-2ylmethyl]-1H-benzimidazole. Arab J Chem 2014;7:418-24

33. Shaharyar M, Mazumder A, Abdullah MM. Synthesis, characterization and antimicrobial activity of $1,3,4$-oxadiazole bearing 1H-benzimidazole derivatives. Arab J Chem 2017;10:S503-8. 